an innovative approach for informing local initiatives and national priorities that has been described as essential and fundamental by city leaders. This presentation will share key components from the largest US initiative focused on cities including, highlighting materials such as the recently updated UNITY RoadMap and discuss lessons learned and their implications for US and international efforts. A UNITY baseline survey of sampled cities showed the majority of cities did not have a plan or coordinating body on violence prevention. In most cities efforts were primarily focused on intervention with little emphasis on prevention. To address these needs and build on whats already working in cities, UNITY has delineated the essential elements for cities to prevent violence via the UNITY RoadMap and an Urban Agenda for Preventing Violence. The UNITY RoadMap builds on similar tools proven effective for other difficult problems including the AIDS Program Efforts Index (developed by US Agency for International Development and United the world against AIDS) and the Urban Agenda details the national support needed to sustain local efforts. In particular, these tools delineate the WHO and WHAT it takes to prevent violence and HOW to maximise and sustain efforts to prevent violence before it occurs. Further, UNITY is coordinating a Network of large cities committed to preventing violence. Network members share successes with each other, strengthen strategy, and call attention to the need for more prevention.

\title{
0643 UNITY: LESSONS LEARNED FROM A NATIONAL INITIATIVE TO PREVENT VIOLENCE
}

L Cohen*, R Davis, D P Stith, B Weiss, H Pinderhuges, A Lyles Correspondence: Prevention Institute, 223 Oak Street Oakland, CA 94607, USA

10.1136/ip.2010.029215.643

Since 2006, Urban Networks to Increase Thriving Youth (UNITY) has worked to support the largest US cities in preventing violence before it occurs. The UNITY Initiative is 